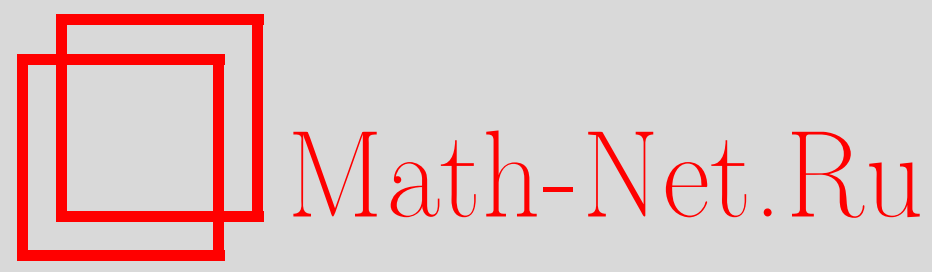

А. П. Старовойтов, K проблеме описания последовательностей наилучших тригонометрических рациональных приближений, Матем. заметки, 2001, том 69, выпуск 6, 919924

DOI: https://doi.org/10.4213/mzm706

Использование Общероссийского математического портала Math-Net.Ru подразумевает, что вы прочитали и согласны с пользовательским соглашением http://www . mathnet.ru/rus/agreement

Параметры загрузки:

IP : 54.172 .240 .79

26 апреля 2023 г., 15:58:00

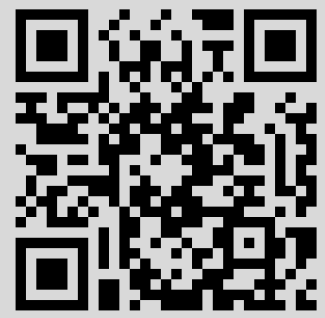




\section{К ПРОБЛЕМЕ ОПИСАНИЯ ПОСЛЕДОВАТЕЛЬНОСТЕЙ НАИЛУЧШИХ ТРИГОНОМЕТРИЧЕСКИХ РАЦИОНАЛЬНЫХ ПРИБЛИЖЕНИЙ}

\section{А. П. Старовойтов}

Для заданной последовательности $\left\{a_{n}\right\}_{n=0}^{\infty}$ неотрицательных действительных чисел, которая строго убывает и сходится к нулю, построена непрерывная $2 \pi$-периодическая функция $f$ такая, что $R_{n}^{T}(f)=a_{n}, n=0,1,2, \ldots$, где $R_{n}^{T}(f)$ - наилучшие приближения функции $f$ тригонометрическими рациональными функциями степени не выше $n$ в равномерной норме.

Библиография: 11 названий.

Пусть $C_{2 \pi}$ - банахово пространство $2 \pi$-периодических непрерывных на всей числовой прямой функций с равномерной нормой. Через $\mathscr{P}_{n}$ и $\mathscr{R}_{n}$ обозначим соответственно множество всех тригонометрических полиномов и тригонометрических рациональных функций с действительными коэффициентами степени не вьше $n$. Для $f \in C_{2 \pi}$ и $n=0,1,2, \ldots$ рассмотрим

$$
E_{n}(f)=\inf \left\{\|f-p\|: p \in \mathscr{P}_{n}\right\}, \quad R_{n}(f)=\inf \left\{\|f-r\|: r \in \mathscr{R}_{n}\right\}
$$

- наилучшие приближения $f$ в $C_{2 \pi}$ множествами $\mathscr{P}_{n}, \mathscr{R}_{n}$.

Хорошо известно, что последовательности $\left\{E_{n}(f)\right\}_{n=0}^{\infty}$ и $\left\{R_{n}(f)\right\}_{n=0}^{\infty}$ не возрастают и сходятся к нулю. Отмеченные свойства наилучших тригонометрических полиномиальных приближений характеризуют их полностью. Именно, справедлива следующая теорема Бернштейна (см. [1], [2]): если последовательность $\left\{a_{n}\right\}_{n=0}^{\infty}$ неотрицательных чисел не возрастает и сходится к нулю, то существует $f \in C_{2 \pi}$, для которой

$$
E_{n}(f)=a_{n}, \quad n=0,1,2, \ldots .
$$

Позже этот результат был перенесен на произвольное банахово пространство (см. [2]).

Аналог теоремы Бернштейна для рациональных приближений отсутствует даже в классическом случае пространства $C_{2 \pi}$, хотя проблема описания всех последовательностей $\left\{R_{n}(f)\right\}_{n=0}^{\infty}, f \in C_{2 \pi}$, поставлена давно (см., например, [3]). По существу, единственньм результатом в этом направлении является следующее утверждение Долженко [3]: для указанной вьше последовательности $\left\{a_{n}\right\}_{n=0}^{\infty}$ существует функция $f \in C_{2 \pi}$ такая, что

$$
R_{9^{k}}(f)=a_{9^{k}}, \quad k=0,1,2, \ldots .
$$

С другой стороны, в случае приближения комплекснозначных функций рациональными функциями с комплексными коэффициентами в решении указанной проблемы имеется существенный сдвиг (см. [4], [5]).

Основным результатом данной работы является следующая 
ТЕОРемА. Для любой строго убывающей к нулю последовательности $\left\{a_{n}\right\}_{n=0}^{\infty}$ неотрицательных чисел существует такая нечетная функция $f \in C_{2 \pi}$, что

$$
R_{n}(f)=a_{n}, \quad n=0,1,2, \ldots
$$

ДокАЗАТЕЛЬСТво теоремы базируется на принципе неподвижной точки Шаудера, а в основу конструкции искомой функции $f \in C_{2 \pi}$ положены алгебраические дроби Чебьшева-Маркова, наименее уклоняющиеся от нуля на отрезке $[-1,1]$. Заметим, что классическое доказательство Бернштейна основано на принципе компактности Больцано-Вейерштрасса, при этом построенная им функция $f \in C_{2 \pi}$ является четной.

Без ограничения общности при построении функции $f$ будем считать, что $a_{0}=1$. В противном случае следует рассмотреть последовательность

$$
1, \frac{a_{1}}{a_{0}}, \frac{a_{2}}{a_{0}}, \ldots
$$

и воспользоваться равенством $R_{n}(\lambda f)=|\lambda| R_{n}(f)$. Определим две бесконечно малые последовательности положительных чисел

$$
\triangle a_{n}=a_{n-1}-a_{n}, \quad \varepsilon_{n}=\min \left\{\triangle a_{1}, \triangle a_{2}, \ldots, \triangle a_{n}\right\}, \quad n=1,2,3 \ldots
$$

Лемма [4]. Существует последовательность $\left\{\beta_{j}\right\}_{j=1}^{\infty}$, удовлетворяющая условиям⿻

$$
\begin{gathered}
1>\beta_{j}>\beta_{j+1}>0, \quad j=1,2,3, \ldots, \\
\sum_{j=l+1}^{\infty} \frac{\beta_{j}}{\beta_{l}}<\frac{1}{4} \varepsilon_{l+1}, \quad l=1,2,3, \ldots, \quad \sum_{j=1}^{l-1} \frac{\beta_{l}}{\beta_{j}}<\frac{1}{4} \varepsilon_{l}, \quad l=2,3,4, \ldots
\end{gathered}
$$

ДокАЗАТЕЛЬСТво. Нужным условиям удовлетворяет последовательность

$$
\beta_{j}=\frac{\varepsilon_{1}}{5} \frac{\varepsilon_{2}}{5} \ldots \frac{\varepsilon_{j}}{5}, \quad j=1,2,3, \ldots
$$

Лемма доказана.

Для каждого $n \in \mathbb{N}$ положим $z_{k}=i \beta_{k}, k=1,2, \ldots, n$, и, взяв каждую точку $z_{k} \mathrm{c}$ кратностью 2, построим синус-дробь Бернштейна [6, гл. $1, \S 1]$

$$
N_{2 n}(y)=\sin \Phi_{2 n}(y)=\frac{y P_{2 n-2}(y)}{\prod_{k=1}^{n}\left(y^{2}+\beta_{k}^{2}\right)},
$$

где $P_{2 n-2}(y)$ - четный полином порядка $2 n-2$, а

$$
\Phi_{2 n}(y)=2 \sum_{k=1}^{n} \arg \left(z_{k}-y\right) .
$$


Лемма 2. Пусть $\lambda_{k, l}=N_{2 k}\left(\beta_{l}\right)$. Тогда

$$
\begin{aligned}
\left|\lambda_{k, l}\right| & <\frac{1}{2} \varepsilon_{l} & \text { nрu } l>k, \\
\left|\lambda_{k, l}-(-1)^{l}\right| & <\frac{1}{2} \varepsilon_{l} & \text { npu } l \leqslant k .
\end{aligned}
$$

ДоКАЗАТЕЛЬСТво. Учитьвая выбор точек $\left\{z_{j}\right\}_{j=1}^{k}$, получим, что при $y>0$

$$
\Phi_{2 k}(y)=2 \sum_{j=1}^{k} \arg \left(i \beta_{j}-y\right)=2 \sum_{j=1}^{k}\left(\frac{\pi}{2}+\operatorname{arctg} \frac{y}{\beta_{j}}\right)=\pi k+2 \sum_{j=1}^{k} \operatorname{arctg} \frac{y}{\beta_{j}} .
$$

Следовательно, если $l>k$, учитьвая лемму 1 , имеем

$$
0<\Phi_{2 k}\left(\beta_{l}\right)-\pi k=2 \sum_{j=1}^{k} \operatorname{arctg} \frac{\beta_{l}}{\beta_{j}} \leqslant 2 \sum_{j=1}^{k} \frac{\beta_{l}}{\beta_{j}}<\frac{1}{2} \varepsilon_{l} .
$$

Отсюда получим

$$
\left|\lambda_{k, l}\right|=\left|\sin \Phi_{2 k}\left(\beta_{l}\right)\right|<\frac{1}{2} \varepsilon_{l},
$$

и неравенство (2) доказано.

Пусть теперь $l \leqslant k$. Тогда из представления

$$
\Phi_{2 k}(y)=2\left\{\sum_{j=1}^{l-1} \arg \left(-y+i \beta_{j}\right)+\arg \left(-y+i \beta_{l}\right)+\sum_{j=l+1}^{k} \arg \left(-y+i \beta_{j}\right)\right\}
$$

имеем, что

$$
\Phi_{2 k}\left(\beta_{l}\right)=\pi(2 k-l)+\frac{\pi}{2}+2 \sum_{j=1}^{l-1} \operatorname{arctg} \frac{\beta_{l}}{\beta_{j}}-2 \sum_{j=l+1}^{k} \operatorname{arctg} \frac{\beta_{j}}{\beta_{l}} .
$$

Снова применив лемму 1 , получим

$$
\begin{aligned}
\left|\Phi_{2 k}\left(\beta_{l}\right)-\pi(2 k-l)-\frac{\pi}{2}\right| & \leqslant 2 \max \left\{\sum_{j=1}^{l-1} \operatorname{arctg} \frac{\beta_{l}}{\beta_{j}}, \sum_{j=l}^{k} \operatorname{arctg} \frac{\beta_{j}}{\beta_{l}}\right\} \\
& <2 \max \left\{\sum_{j=1}^{l-1} \frac{\beta_{l}}{\beta_{j}}, \sum_{j=l}^{k} \frac{\beta_{j}}{\beta_{l}}\right\}<\frac{1}{2} \varepsilon_{l} .
\end{aligned}
$$

Отсюда следует неравенство (3). Лемма доказана.

Сделав в (1) замену $x=\left(1-y^{2}\right) /\left(1+y^{2}\right)$, придем к синус-дроби Чебьшева-Маркова, которая имеет следующий вид $[6$, гл. $2, \S 1$, равенство (9)]:

$$
\nu_{n}(x)=\sin \varphi_{2 n}(x)=\sin \Phi_{2 n}(y)=\sqrt{1-x^{2}} \frac{P_{n-1}(x)}{\prod_{j=1}^{n}\left(1-c_{j} x\right)},
$$


где $P_{n-1}(x)$ - алгебраический полином степени $n-1$ с действительными коэффициентами, а $c_{k}=\left(1-\beta_{k}^{2}\right) /\left(1+\beta_{k}^{2}\right), k=1,2, \ldots, n$. С учетом замены и последних равенств получим, что $0<c_{1}<c_{2}<\cdots<c_{n}<\cdots<1$ и $\nu_{k}\left(c_{l}\right)=\lambda_{k, l}, k=1,2, \ldots, l=1,2, \ldots$

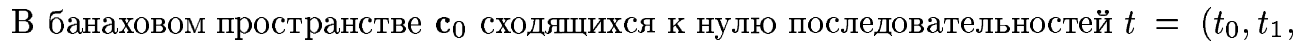
$\left.t_{2}, \ldots\right)$ с нормой $\|t\|_{c_{0}}=\sup \left\{\left|t_{n}\right|: n=0,1,2, \ldots\right\}$ определим выпуклое компактное множество

$$
K=\left\{t: 0 \leqslant t_{k} \leqslant \varepsilon_{k+1} ; \quad k=0,1,2, \ldots\right\} .
$$

Если $t=\left(t_{0}, t_{1}, t_{2}, \ldots\right) \in K$, то полагая $\triangle t_{k}=t_{k-1}-t_{k}$, рассмотрим функцию

$$
f_{t}(\theta)=\sum_{k=1}^{\infty}\left(\triangle a_{k}+\triangle t_{k}\right) \sin \theta \frac{P_{k-1}(\cos \theta)}{\prod_{j=1}^{k}\left(1-c_{j} \cos \theta\right)} .
$$

Поскольку ряд (4) равномерно сходится на любом конечном отрезке, то $f_{t}(\theta)$ является нечетной функцией из $C_{2 \pi}$. Покажем, что семейство функций $\left(f_{t}(\theta)\right)_{t \in K}$ содержит функцию $f$ с нужными свойствами.

Лемма 3. Пусть $\theta_{k}=\arccos c_{k}, k=1,2, \ldots$ Тогда $0<\theta_{k}<\pi / 2$ и при всех $t \in K$ справедливы неравенства

$$
(-1)^{l} f_{t}\left(\theta_{l}\right) \geqslant a_{l-1}+t_{l-1}-\varepsilon_{l}, \quad l=1,2, \ldots .
$$

ДоКАЗАТЕЛЬСТВо. Заметим, что при $\theta \in[0, \pi / 2]$

$$
\sin \theta \frac{P_{k-1}(\cos \theta)}{\prod_{j=1}^{k}\left(1-c_{j} \cos \theta\right)}=\nu_{k}(\cos \theta) .
$$

С учетом этого равенства имеем

$$
\begin{aligned}
f_{t}\left(\theta_{l}\right)-(-1)^{l}\left(a_{l-1}+t_{l-1}\right) & =\sum_{k=1}^{\infty}\left(\triangle a_{k}+\triangle t_{k}\right) \nu_{k}\left(c_{l}\right)-(-1)^{l}\left(a_{l-1}+t_{l-1}\right) \\
& =\sum_{k=1}^{l-1}\left(\triangle a_{k}+\triangle t_{k}\right) \lambda_{k, l}+\sum_{k=l}^{\infty}\left(\triangle a_{k}+\triangle t_{k}\right)\left(\lambda_{k, l}-(-1)^{l}\right) .
\end{aligned}
$$

Поскольку $t \in K$, то $\triangle a_{k}+\Delta t_{k} \geqslant 0$. Поэтому, используя лемму 2 , получим

$$
\left|f_{t}\left(\theta_{k}\right)-(-1)^{l}\left(a_{l-1}+t_{l-1}\right)\right| \leqslant \frac{1}{2} \varepsilon_{l} \sum_{k=1}^{\infty}\left(\triangle a_{k}+\triangle t_{k}\right) \leqslant \frac{1}{2} \varepsilon_{l}\left(a_{0}+t_{0}\right)<\varepsilon_{l} .
$$

Отсюда следует (5). Лемма доказана.

Перейдем теперь к доказательству теоремы. Для этого заметим, что при любом $t \in K$ функция $f_{t}$ подчинена условиям

$$
a_{n}+t_{n}-\varepsilon_{n+1} \leqslant R_{n}\left(f_{t}\right) \leqslant a_{n}+t_{n}, \quad n=0,1,2, \ldots
$$

Действительно, так как $\left|\nu_{k}(x)\right|=\left|\sin \varphi_{2 k}(x)\right| \leqslant 1$ и $\triangle a_{k}+\Delta t_{k} \geqslant 0$, то

$$
\begin{aligned}
& \left|f_{t}(\theta)-\sum_{k=1}^{n}\left(\triangle a_{k}+\triangle t_{k}\right) \sin \theta \frac{P_{k-1}(\cos \theta)}{\prod_{j=1}^{k}\left(1-c_{j} \cos \theta\right)}\right| \\
& \quad \leqslant \sum_{k=n+1}^{\infty}\left(\triangle a_{k}+\triangle t_{k}\right)\left|\nu_{k}(\cos \theta)\right| \leqslant \sum_{k=n+1}^{\infty}\left(\triangle a_{k}+\triangle t_{k}\right)=a_{n}+t_{n} .
\end{aligned}
$$

Таким образом, оценка сверху в (6) доказана. Для доказательства оценки снизу воспользуемся следующим аналогом теоремы Валле-Пуссена $[7$, гл. 2, § 31] для периодического случая. 
Лемма 4. Если $f \in C_{2 \pi}$ и принимает в последовательных точках $u_{1}<u_{2}<\cdots$ $\cdots<u_{N}$ промежутка $(-\pi, \pi]$ отличные от нуля значения $\lambda_{1},-\lambda_{2}, \ldots,(-1)^{N-1} \lambda_{N}$ с чередуюшимися знаками (все $\lambda_{i}$ имеют одинаковый знак), причем $N \geqslant 2 n+2$, mo

$$
R_{n}(f) \geqslant \min \left\{\lambda_{1}, \lambda_{2}, \ldots, \lambda_{N}\right\}
$$

На промежутке $(-\pi, \pi]$ имеется с учетом нечетности функции $f_{t}(\theta) 2 n+2$ точек $u_{1}=\theta_{1}, u_{2}=\theta_{2}, \ldots, u_{n+1}=\theta_{n+1}, u_{n+2}=-\theta_{n+1}, u_{n+3}=-\theta_{n}, \ldots, u_{2 n+2}=-\theta_{1}$, в которых согласно (5) функция $f_{t}$ принимает значения с чередующимися знаками. Поэтому из лемм 3,4 получим оценку снизу в (6). Неравенства (6) означают, что отображение $\Pi: \mathbf{c}_{0} \rightarrow \mathbf{c}_{0}$, определяемое равенством

$$
\Pi(t)=\left\{a_{n}+t_{n}-R_{n}\left(f_{t}\right)\right\}_{n=0}^{\infty},
$$

переводит $K$ в себя.

Лемма 5. Отображсение $t \mapsto f_{t}(\theta)$ банахова пространства с в $_{0} C_{2 \pi}$ равномерно непрерывно на $K$.

ДокАЗАТЕЛЬСТво. Пусть $t^{\prime}, t^{\prime \prime} \in K$. Тогда с учетом (4) будем иметь, что

$$
\begin{aligned}
\max _{\theta \in \mathbb{R}}\left|f_{t^{\prime \prime}}(\theta)-f_{t^{\prime}}(\theta)\right| & \leqslant \sum_{k=1}^{N}\left|\Delta t_{k}^{\prime \prime}-\Delta t_{k}^{\prime}\right|+\sum_{k=N+1}^{\infty}\left(\Delta a_{k}+\Delta t_{k}^{\prime \prime}\right)+\sum_{k=N+1}^{\infty}\left(\triangle a_{k}+\triangle t_{k}^{\prime}\right) \\
& \leqslant 2 N \max _{0 \leqslant k \leqslant N}\left|t_{k}^{\prime \prime}-t_{k}^{\prime}\right|+2 a_{N}+t_{N}^{\prime \prime}+t_{N}^{\prime} .
\end{aligned}
$$

Отсюда и вытекает утверждение леммы. Лемма доказана.

ЛЕмма 6. Пусть $f, g \in C_{2 \pi}$ и для некоторого $\varepsilon>0$ верно неравенство $\|f-g\|<\varepsilon$. Тогда

$$
\left|R_{n}(f)-R_{n}(g)\right|<\varepsilon \quad \partial \text { я } n=0,1,2, \ldots
$$

ДокАЗАТЕЛЬСТВо. Пусть $r_{n}^{f}$ и $r_{n}^{g}$-тригонометрические рациональные функции наилучшего приближения $f$ и $g$ степени не выше $n$. Тогда из условия леммы следует, что

$$
\left\|f-r_{n}^{g}\right\|<R_{n}(g)+\varepsilon, \quad\left\|g-r_{n}^{f}\right\|<R_{n}(f)+\varepsilon
$$

поэтому

$$
R_{n}(f)<R_{n}(g)+\varepsilon, \quad R_{n}(g)<R_{n}(f)+\varepsilon
$$

Лемма доказана.

Из леммы 5 и 6 следует, что отображение П $(t)$ непрерьвно на $K$. По теореме Шаудера (см. [8]) существует точка $t^{*}=\left(t_{0}^{*}, t_{1}^{*}, t_{2}^{*}, \ldots\right) \in K$, для которой $\Pi\left(t^{*}\right)=t^{*}$, т.е. $R_{n}\left(f_{t^{*}}\right)=a_{n}, n=0,1,2, \ldots$ Следовательно, функция $f(\theta)=f_{t^{*}}(\theta)$ является искомой. Теорема доказана.

Отметим, что приведенное доказательство теоремы в сравнении с доказательством Бернштейна является более конструктивным. В частности, задав нужную скорость 
убывания к нулю последовательности $\left\{a_{k}\right\}_{n=0}^{\infty}$, можно изучать различные свойства всего семейства функций $\left(f_{t}(\theta)\right)_{t \in K}$, которому принадлежит искомая функция. Данное обстоятельство имеет важное значение как при доказательстве точности прямых теорем рациональной аптроксимации, так и при получении обратных теорем.

Основные результаты статьи анонсированы в [9]-[11].

В заключение автор выражает благодарность профессору В. Н. Русаку и профессору А. А. Пекарскому за полезные обсуждения полученных результатов и внимание к работе.

\section{СПИСОК ЦИТИРОВАННОЙ ЛИТЕРАТУРЫ}

[1] Натансон И. П. Конструктивная теория функций. М.-Л.: ГИТТЛ, 1949.

[2] Тиман А.Ф. Теория приближения функций действительного переменного. М.: ГИФМЛ, 1950.

[3] Долженко Е. П. Сравнение скоростей рациональной и полиномиальной аппроксимаций // Матем. заметки. 1967. Т. 1. №3. С. 313-320.

[4] Пекарский А. А. Существование функции с заданными наилучшими равномерными рациональными приближениями // Изв. АН Беларуси. Сер. физ. -матем. науки. 1994. № 1. С. 23-26.

[5] Назаренко М. А. Существование функции с заданными рациональными приближениями в пространстве $C(A) / /$ Вестн. МГУ. Сер. 1. Матем., мех. 1997. №4. С. 20-22.

[6] Русак В. Н. Рациональные функции как аппарат приближения. Минск: БГУ, 1979.

[7] Ахиезер Н. И. Лекции по теории аппроксимации. М.: Наука, 1965.

[8] Канторович Л.В., Акилов Г. П. Функциональный анализ. М.: Наука, 1977.

[9] Старовойтов А.П. Об одной проблеме Бернштейна-Долженко // International Conference on Approximation Theory and its Applications Dedicated to the Memory of V. K. Dzjadyk. Abstracts. Kyiv: Institute of Math. Nat. Acad. Sci. Ukraine, 1999. P. 78.

[10] Старовойтов А. П. Описание последовательостей наилучших тригонометрических рациональных приближений // Аналитические методы анализа и дифференциальных уравнений. Тез. докл. междунар. конф. (14-18 сентебря 1999г., Минск, Беларусь). Минск: БГУ, 1999. С. $213-214$.

[11] Старовойтов А. П. К проблеме описания последовательностей наилучших тригонометрических рациональных приближений // Докл. НАН Беларуси. 2000. Т. 44. № 2. С. 23-25.

Гомельский государственный университет им. $\Phi$. Скорины

Поступило E-mail: svoitov@gsu.unibel.by 